\title{
Bean quality traits and sensory evaluation of wild Guianan cocoa populations (Theobroma cacao L.)
}

\author{
S. Assemat $\cdot$ Ph. Lachenaud $\cdot$ F. Ribeyre $\cdot$ \\ F. Davrieux $\cdot$ J.-L. Pradon $\cdot$ E. Cros
}

Received: 11 July 2003/ Accepted: 28 November 2003/Published online: 23 September 2006

(C) Springer Science+Business Media B.V. 2006

\section{Erratum to: Genet Resour Crop Evol: 52: 911- 917 DOI 10.1007/s10722-003-6117-2}

In Genet Resour Crop Evol (2005) 52: 911-917; Throughout the article 'naturalized cocoa' should be replaced by 'wild cocoa'. In the Reference section, Lanaud et al. reference should read 'Lanaud C., Motamayor J.-C. and Sounigo O. 1999. Le cacaoyer. In: Diversité génétique des plantes tropicales cultivées. Hamon P., Seguin M., Perrier X., Glaszmann J.-C (eds), Collection Repères, CIRAD, 387 p.'

The online version of the original article can be found at http://dx.doi.org/10.1007/s10722-003-6117-2

S. Assemat $\cdot \mathrm{Ph}$. Lachenaud $(\bowtie) \cdot$ F. Ribeyre

F. Davrieux · J.-L. Pradon · E. Cros

CIRAD-CP, 34398 Montpellier Cedex 5, France

e-mail: lachenaud@cirad.fr

J.-L. Pradon

CIRAD-CP, BP 701, 97397 Kourou, France 